Research Article

\title{
Effect of Geometric Nonlinearity on Membrane Roof Stability in Air Flow
}

\author{
Weiju Song $\left(\mathbb{D},{ }^{1}\right.$ Jun $\mathrm{Xu}^{2}{ }^{2}$ Xiaowei Wang $\left(\mathbb{D},{ }^{2}\right.$ and Changjiang Liu $\mathbb{D}^{3}$ \\ ${ }^{1}$ College of Civil Engineering, Hebei University of Engineering, Handan 056038, China \\ ${ }^{2}$ School of Civil Engineering, Chongqing University, Chongqing 400045, China \\ ${ }^{3}$ School of Civil Engineering, Guangzhou University, Guangzhou 510006, China \\ Correspondence should be addressed to Weiju Song; nimrodsong@126.com
}

Received 20 August 2019; Revised 30 December 2019; Accepted 7 January 2020; Published 9 April 2020

Academic Editor: Franck Poisson

Copyright (C) 2020 Weiju Song et al. This is an open access article distributed under the Creative Commons Attribution License, which permits unrestricted use, distribution, and reproduction in any medium, provided the original work is properly cited.

Membrane materials are widely used in construction engineering with small mass and high flexibility, which presents strong geometric nonlinearity in vibration. In this paper, an improved multiscale perturbation method is used to solve the aerostatics stability of membrane roofs on closed and open structures by quantifying the effect of geometric nonlinearity on the single-mode aeroelastic instability wind velocity. Results show that the critical wind velocities of two models are smaller when the geometrical nonlinearity of the membrane material is neglected. In addition, under normal wind load, the influence of geometrical nonlinearity of the membrane on the aerodynamic stability of the roof can be neglected. However, under strong wind load, when the roof deformation reaches $3 \%$ of the span, the influence of geometric nonlinearity should be considered and the influence increases with the decrease of transverse and downwind span of the membrane roof. The results obtained in this paper have an important theoretical reference value for the design membrane structures.

\section{Introduction}

Fabric membrane is a widely used membrane material in construction engineering. It has the characteristics of high tensile strength and good flexibility. Fabric membranes are mainly composed of substrates and coatings. The substrates are usually braided by orthogonal fibers, which results in the orthotropic properties of the membranes. That is to say, the elastic modulus and Poisson's ratio in the two orthogonal directions are different. The building, which is made up of membrane material covered on the structural skeleton or tensioned as a whole, has beautiful appearance, good transparency, environmental protection, and energy saving $[1,2]$. Therefore, it is widely used in large-scale stadiums, exhibition venues, and other public buildings. Because of the small mass and flexibility, it is easy for vibration under external disturbance, and the stiffness of the membrane material is small, which results in the large vibration deformation of the membrane structure under wind load, showing strong geometric nonlinearity [3, 4]. Many research results show that the single-mode aeroelastic instability can easily occur in membrane structures when the pretension of membrane materials is small $[5,6]$.

In the mathematical analysis of aeroelastic instability of flexible membrane structures, Yang and Liu $[7,8]$ established the wind-induced dynamic coupling equation of hyperbolic parabolic membrane roof with small sag by using elastic shallow shell theory and ideal fluid potential flow theory in 2006 and determined the critical wind velocity of aeroelastic instability according to Routh-Hurwitz stability criterion. The influence of geometric nonlinearity of membranes was not considered when establishing the mathematical model. In 2011, Xu et al. [9, 10] studied the nonlinear aerodynamic stability of orthotropic-tensioned membrane structures in a rectangular plane and hyperbolic paraboloid, respectively. The critical wind velocities of single-mode instability of the two membrane structures were determined by assuming the solution of the vibration equation. In 2017, Liu et al. [11] studied the aerodynamic stability of closed-tensioned membrane structures by the 
Galerkin method. The geometric nonlinearity of membrane vibration is weakened, and the critical wind velocity of the instability is obtained by using the weak nonlinearity solution method.

In order to investigate the influence of the geometric nonlinearity on the aeroelastic stability of membrane materials, the nonlinear wind-induced dynamic equations of membrane roofs are established based on Von Kamen's large deflection theory and Darumbel's principle, taking the flat rectangular orthotropic tensioned membrane roofs with fixed supports on the four sides of open and closed structures as analytical models and considering the effects of geometric nonlinearity and air damping of membrane materials. An improved multiscale method which is suitable for strong geometric nonlinearity is used to solve the vibration equation. The critical wind velocity of instability obtained is compared with the results without considering geometric nonlinearity. The effect of geometric nonlinearity on the wind velocity of singlemode aeroelastic instability of membrane material is obtained quantitatively.

\section{Analytical Deduction of Single-Mode Instability of Orthotropic Membrane Roofs}

2.1. Establishment of Basic Equations. Let the length and width of the orthotropic rectangular flexible membrane with four sides fixed be $a$ and $b$, respectively; the pretension along the length direction is $N_{0 x}$ and the width direction is $N_{0 y}$. The wind blows parallel to the roof and toward the membrane surface, which makes the membrane surface vibrate. For flexible membranes, the research results show that the shear stress has little influence on the vibration process of the membranes and can be considered as zero [9-13]. Assuming that the planar membrane is in the XOY plane when in equilibrium and the pretension in $X$ direction is $N_{x}$ and in the $Y$ direction is $N_{y}$, when the membrane is disturbed by external forces to the XOY plane, it will deform and then produce transverse vibration perpendicular to the membrane surface under the action of tension. Select a microunit on the vibrating membrane surface, as shown in Figure 1.

Select a unit part dxdy on the membrane surface. When the microunits are deformed, the edges of the microunits are subjected to the tension of the adjacent facets. In $X$ direction, we can regard the surface element as composed of countless chord elements with length $\mathrm{d} x$ and width of one unit. The tension acting on the chord element is consistent with its tangent direction. The tension $N_{x}$ is at an angle $\alpha$ with $X$ coordinate axis. Therefore, the vertical component of the tension acting on the chord element at one end of $X$ is $N_{x} \sin \alpha$. Because $\alpha$ is small, $\sin \alpha \approx \tan \alpha$. Let $w$ be the vertical displacement of a point on the membrane away from the equilibrium position. Therefore,

$$
N_{x} \sin \alpha=N_{x} \tan \alpha=N_{x}\left(\frac{\partial w}{\partial x}\right)_{x} .
$$

The vertical force acting on the $\mathrm{d} y$ edge is $N_{x}(\partial w / \partial x)_{x}$ $\mathrm{d} y$, and the vertical force at the $x$ edge should be

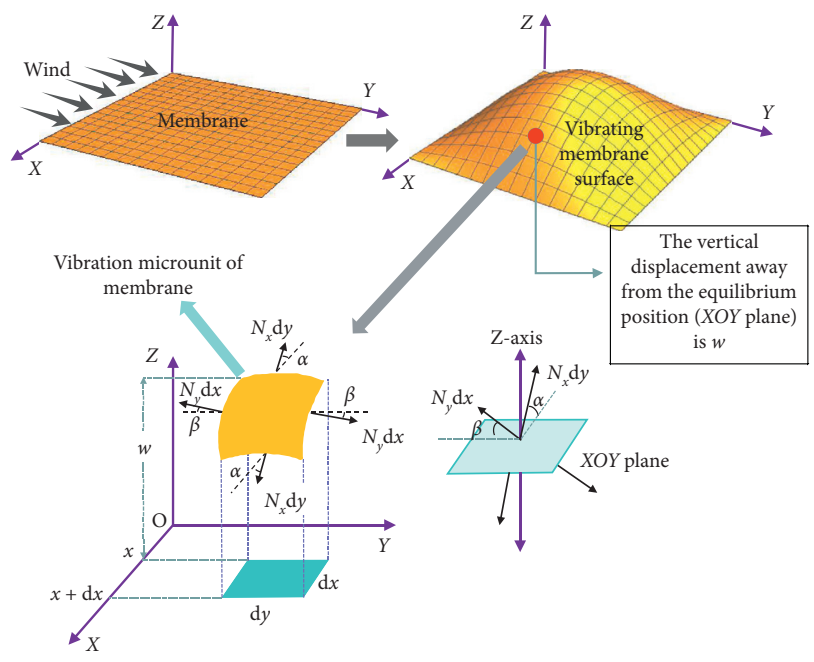

FiguRE 1: Vibration microunits of membrane.

$N_{x}(\partial w / \partial x)_{x+\mathrm{d} x} \mathrm{~d} y$. Thus, the resultant force in $Z$ direction on the $x$ and $x+\mathrm{d} x$ sides of the panel is as follows:

$$
N_{x}\left(\frac{\partial w}{\partial x}\right)_{x+\mathrm{d} x} \mathrm{~d} y-N_{x}\left(\frac{\partial w}{\partial x}\right)_{x} \mathrm{~d} y=N_{x} \frac{\partial^{2} w}{\partial x^{2}} \mathrm{~d} x \mathrm{~d} y .
$$

Similarly, the resultant force of $Z$ direction component of the tension acting on $Y$ direction can be obtained as follows:

$$
N_{y}\left(\frac{\partial w}{\partial y}\right)_{y+\mathrm{d} y} \mathrm{~d} x-N_{y}\left(\frac{\partial w}{\partial y}\right)_{y} \mathrm{~d} x=N_{y} \frac{\partial^{2} w}{\partial y^{2}} \mathrm{~d} x \mathrm{~d} y .
$$

So the total force in $Z$ direction acting on the whole panel is

$$
F_{z}=N_{x} \frac{\partial^{2} w}{\partial x^{2}} \mathrm{~d} x \mathrm{~d} y+N_{y} \frac{\partial^{2} w}{\partial y^{2}} \mathrm{~d} x \mathrm{~d} y+q(x, y) \mathrm{d} x \mathrm{~d} y,
$$

where $N_{x}$ is the tension in $X$ direction (longitude), $N_{y}$ is the tension in $Y$ direction (latitude), $w$ is the deflection of the membrane, and $q(x, y)$ is the external load acting on the unit area of the projection surface of the membrane. According to the force balance, we can obtain that

$$
\begin{array}{r}
N_{x} \frac{\partial^{2} w}{\partial x^{2}} \mathrm{~d} x \mathrm{~d} y+N_{y} \frac{\partial^{2} w}{\partial y^{2}} \mathrm{~d} x \mathrm{~d} y+q(x, y) \mathrm{d} x \mathrm{~d} y=0 \\
q(x, y)+N_{x} \frac{\partial^{2} w}{\partial x^{2}}+N_{y} \frac{\partial^{2} w}{\partial y^{2}}=0 .
\end{array}
$$

The generalized external loads of the flexible membrane roof under wind load include the wind load acting on the membrane surface, structural damping force, and inertial force [11]. If the aerodynamic term is defined as $p$, then the generalized external load per unit area $q(x, y)$ is

$$
q(x, y)=p(x, y, t)-2 \rho c \frac{\partial w(x, y, t)}{\partial t}-\rho \frac{\partial^{2} w(x, y, t)}{\partial t^{2}} .
$$

For membrane material, the stiffness of the membrane surface comes from the initial pretension of membrane 
material, so the initial pretension should be added in equation (6). Finally, the differential equations of motion of flexible membranes are obtained as follows:

$$
\begin{aligned}
& p(x, y, t)-2 \rho c \frac{\partial w(x, y, t)}{\partial t}-\rho \frac{\partial^{2} w(x, y, t)}{\partial t^{2}} \\
& +\left(N_{0 x}+N_{x t}\right) \frac{\partial^{2} w}{\partial x^{2}}+\left(N_{0 y}+N_{y t}\right) \frac{\partial^{2} w}{\partial y^{2}}=0 .
\end{aligned}
$$

Introducing the stress function $\varphi(x, y), N_{x}=h\left(\partial^{2} \varphi /\right.$ $\left.\partial y^{2}\right)$ and $N_{y}=h\left(\partial^{2} \varphi / \partial x^{2}\right)$. Then, equation (6) can be transformed as

$$
\left(N_{0 x}+h \frac{\partial^{2} \varphi}{\partial y^{2}}\right) \frac{\partial^{2} w}{\partial x^{2}}+\left(N_{0 y}+h \frac{\partial^{2} \varphi}{\partial x^{2}}\right) \frac{\partial^{2} w}{\partial y^{2}}+p-2 \rho c \frac{\partial w}{\partial t}-\rho \frac{\partial^{2} w}{\partial t^{2}}=0 .
$$

After deformation, the membrane surface strain is composed of linear and nonlinear parts. The linear strain is caused by in-plane displacement $u$ and $v$, and the nonlinear strain is caused by deflection $w$. After ignoring shear stress, the total strain is as follows:

$$
\left.\begin{array}{l}
\varepsilon_{x}=\frac{\partial u}{\partial x}+\frac{1}{2}\left(\frac{\partial w}{\partial x}\right)^{2} \\
\varepsilon_{y}=\frac{\partial v}{\partial y}+\frac{1}{2}\left(\frac{\partial w}{\partial y}\right)^{2}
\end{array}\right\}
$$

where $\varepsilon_{x}$ is the strain in $X$ direction and $\varepsilon_{y}$ is the strain in $Y$ direction.

By eliminating $u$ and $v$ in equation (10), the continuous deformation conditions satisfying the strain and deflection of the membrane surface can be obtained:

$$
\frac{\partial^{2} \varepsilon_{x}}{\partial y^{2}}+\frac{\partial^{2} \varepsilon_{y}}{\partial x^{2}}=\left(\frac{\partial^{2} w}{\partial x \partial y}\right)^{2}-\frac{\partial^{2} w}{\partial x^{2}} \frac{\partial^{2} w}{\partial y^{2}} .
$$

The membrane is orthotropic, and the direction of the fiber is the main direction of elasticity so that it is consistent with the direction of coordinate system $X$ and $Y$. Assuming that the direction of fiber is the same as the direction of coordinate system $X$ and $Y$, Young's modulus of elasticity in $X$ and $Y$ directions is $E_{1}$ and $E_{2}$, respectively. The longitudinal Poisson's ratio and the latitudinal Poisson's ratio is $\mu_{1}$ and $\mu_{2}$, respectively. The relationship between elastic modulus and Poisson's ratio is as follows:

$$
\frac{\mu_{1}}{E_{1}}=\frac{\mu_{2}}{E_{2}}
$$

The stress-strain relationship is as follows:

$$
\left\{\begin{array}{c}
\sigma_{x} \\
\sigma_{y}
\end{array}\right\}=\left(\begin{array}{cc}
\frac{E_{1}}{1-\mu_{1} \mu_{2}} & \frac{\mu_{1} E_{2}}{1-\mu_{1} \mu_{2}} \\
\frac{\mu_{2} E_{1}}{1-\mu_{1} \mu_{2}} & \frac{E_{2}}{1-\mu_{1} \mu_{2}}
\end{array}\right)\left\{\begin{array}{c}
\varepsilon_{x} \\
\varepsilon_{y}
\end{array}\right\},
$$

where $\sigma_{x}$ and $\sigma_{y}$ is the normal stresses in $X$ and $Y$ directions, respectively. $h$ is the thickness of membrane.
Letting $N_{x}=h \cdot \sigma_{x}$ and $N_{y}=h \cdot \sigma_{y}$ and substituting equation (13) into equation (11), the compatibility equation is obtained as

$$
\begin{aligned}
& \frac{1}{E_{1} h} \frac{\partial^{2} N_{x}}{\partial y^{2}}-\frac{\mu_{2}}{E_{2} h} \frac{\partial^{2} N_{y}}{\partial y^{2}}-\frac{\mu_{1}}{E_{1} h} \frac{\partial^{2} N_{x}}{\partial x^{2}} \\
& +\frac{1}{E_{2} h} \frac{\partial^{2} N_{y}}{\partial x^{2}}=\left(\frac{\partial^{2} w}{\partial x \partial y}\right)^{2}-\frac{\partial^{2} w}{\partial x^{2}} \frac{\partial^{2} w}{\partial y^{2}}
\end{aligned}
$$

By substituting the stress function into equation (14), then (14) can be transformed as

$$
\begin{gathered}
\frac{1}{E_{1}} \frac{\partial^{4} \varphi}{\partial y^{4}}-\frac{\mu_{2}}{E_{2}} \frac{\partial^{4} \varphi}{\partial x^{2} \partial y^{2}}-\frac{\mu_{1}}{E_{1}} \frac{\partial^{4} \varphi}{\partial x^{2} \partial y^{2}} \\
+\frac{1}{E_{2}} \frac{\partial^{4} \varphi}{\partial x^{4}}=\left(\frac{\partial^{2} w}{\partial x \partial y}\right)^{2}-\frac{\partial^{2} w}{\partial x^{2}} \frac{\partial^{2} w}{\partial y^{2}} .
\end{gathered}
$$

2.2. Improved Multiscale Solutions of Governing Equations. The initial surface function of the rectangular planar membrane $z_{0}(x, y)=0$. Therefore, the surface equation of the flexible membrane under wind load is as follows:

$$
z(x, y, t)=w(x, y, t)
$$

According to the Bubnov-Galerkin method, assume the solution of the governing equation is [9-12]

$$
\left\{\begin{array}{l}
w(x, y, t)=\sum_{i=1}^{n} T_{i}(t) W_{i}(x, y), \\
\varphi(x, y, t)=\sum_{i=1}^{n} U_{i}(t) \phi_{i}(x, y),
\end{array}\right.
$$

where $W_{i}(x, y)$ is the mode function, $\phi_{i}(x, y)$ is the unknown stress function, and $T_{i}(t)$ and $U_{i}(t)$ are time-dependent functions.

Because the membrane is fixed on four sides, the vertical deflection at the boundary of the membrane is zero, and the vibration mode function satisfying the conditions is assumed to be $[11,12]$

$$
W(x, y)=\sin \frac{m \pi x}{a} \sin \frac{n \pi y}{b},
$$

where $m$ and $n$ are positive integer.

Substituting equation (18) into equation (17), the following equation can be obtained:

$$
w(x, y, t)=T(t) \sin \frac{m \pi x}{a} \sin \frac{n \pi y}{b} .
$$

Substituting equation (19) into equation (15) yields

$$
\frac{1}{E_{1}} \frac{\partial^{4} \varphi}{\partial y^{4}}+\frac{1}{E_{2}} \frac{\partial^{4} \varphi}{\partial x^{4}}=\frac{m^{2} n^{2} \pi^{4}}{2 a^{2} b^{2}} T^{2}(t)\left(\cos \frac{2 m \pi x}{a}+\cos \frac{2 n \pi y}{b}\right) .
$$

Assume that the solution of the stress function in equation (20) is [12] 


$$
\left\{\begin{array}{l}
\varphi(x, y, t)=T^{2}(t) \phi(x, y) \\
\phi(x, y)=\alpha \cos \frac{2 m \pi x}{a}+\beta \cos \frac{2 n \pi y}{b} .
\end{array}\right.
$$

Substituting equation (21) into equation (20) yields

$$
\begin{aligned}
& \alpha=\frac{E_{2} a^{2} n^{2}}{32 b^{2} m^{2}}, \\
& \beta=\frac{E_{1} b^{2} m^{2}}{32 a^{2} n^{2}} .
\end{aligned}
$$

2.2.1. Solution of Flexible Membrane Roof on Closed Structure. Flexible membrane covers the top of the closed structure as the roof and the rigid wall around the structure as the vertical bearing member. Because its stiffness is far greater than the membrane's stiffness, it is assumed that the stiffness of the vertical component is infinite in the process of theoretical derivation in this paper. The membrane roof on closed structure is shown in Figure 2.

For the membrane roof on closed structure, the aerodynamic force acting on the unit area of the membrane projection surface is expressed as [10]

$$
p=-\frac{\rho_{0}}{2 \pi}\left(\begin{array}{c}
(V(\partial w / \partial x)+(\partial w / \partial t)) \begin{array}{c}
(x-\xi) \\
y=\eta
\end{array}-V \iint_{R a} \frac{\left(V\left(\partial^{2} w / \partial x \partial t\right)+\left(\partial^{2} w / \partial t^{2}\right)\right) x=\xi}{\left(\sqrt{(x-\xi)^{2}+(y-\eta)^{2}}\right)^{3}} \mathrm{~d} \xi \mathrm{d} \eta+\iint_{R a} \frac{y=\eta}{\sqrt{(x-\xi)^{2}+(y-\eta)^{2}}} \mathrm{~d} \xi \mathrm{d} \eta
\end{array}\right)
$$

Substituting equation (23) into equation (9) yields

$$
\begin{aligned}
& \left(h \frac{\partial^{2} \varphi}{\partial y^{2}}+N_{0 x}\right) \frac{\partial^{2} w}{\partial x^{2}}+\left(h \frac{\partial^{2} \varphi}{\partial x^{2}}+N_{0 y}\right) \frac{\partial^{2} w}{\partial y^{2}}-2 \rho c \frac{\partial w}{\partial t} \\
& -\rho \frac{\partial^{2} w}{\partial t^{2}}-\frac{\rho_{0}}{\pi} \iint_{R a} \frac{1}{r}\left(\frac{\partial^{2} w}{\partial t^{2}}\right) \begin{aligned}
x & =\xi \\
y & =\eta
\end{aligned} \quad \mathrm{d} \xi \mathrm{d} \eta-\frac{\rho_{0} V}{2 \pi} \iint_{R a} \frac{1}{r}\left(\frac{\partial^{2} w}{\partial x \partial t}\right) \underset{x}{x}=\xi \mathrm{d} \xi \mathrm{d} \eta
\end{aligned}
$$

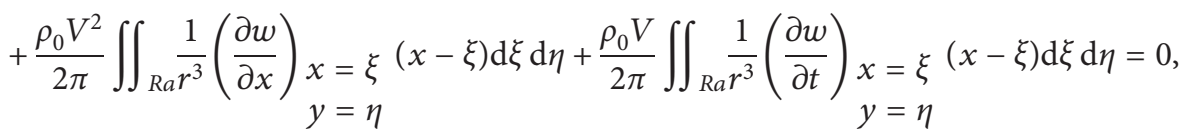

where $r=\sqrt{(x-\xi)^{2}+(y-\eta)^{2}}$ and the integral region $R a \in\{0 \leq \xi \leq a, 0 \leq \eta \leq b\}$.
Substituting equations (19), (21), and (22) into equation (24) yields

$$
\begin{aligned}
& \gamma_{1}=\iint_{R a} \frac{1}{r}(W)_{x=\xi} \mathrm{d} \xi \mathrm{d} \eta=\iint_{R a} \frac{1}{r} \sin \frac{m \pi \xi}{a} \sin \frac{n \pi \eta}{b} \mathrm{~d} \xi \mathrm{d} \eta, \\
& y=\eta \\
& \gamma_{2}=\iint_{R a} \frac{1}{r}\left(\frac{\partial W}{\partial x}\right) x=\xi \mathrm{d} \xi \mathrm{d} \eta=\frac{m \pi}{a} \iint_{R a} \frac{1}{r} \cos \frac{m \pi \xi}{a} \sin \frac{n \pi \eta}{b} \mathrm{~d} \xi \mathrm{d} \eta, \\
& y=\eta \\
& \gamma_{3}=\iint_{R a} \frac{1}{r^{3}}\left(\frac{\partial W}{\partial x}\right) \begin{array}{l}
x=\xi \\
y=\eta
\end{array}(x-\xi) \mathrm{d} \xi \mathrm{d} \eta=\frac{m \pi}{a} \iint_{R a} \frac{1}{r^{3}}(x-\xi) \cos \frac{m \pi \xi}{a} \sin \frac{n \pi \eta}{b} \mathrm{~d} \xi \mathrm{d} \eta, \\
& \gamma_{4}=\iint_{R a} \frac{1}{r^{3}}(W)_{x=\xi}(x-\xi) \mathrm{d} \xi \mathrm{d} \eta=\iint_{R a} \frac{1}{r^{3}}(x-\xi) \sin \frac{m \pi \xi}{a} \sin \frac{n \pi \eta}{b} \mathrm{~d} \xi \mathrm{d} \eta . \\
& y=\eta
\end{aligned}
$$


By using the Bubnov-Galerkin method, (25) can be integrated as

$$
\begin{aligned}
& \iint_{S}\left\{\rho W+\frac{\rho_{0}}{\pi} \gamma_{1} \frac{\mathrm{d}^{2} T t}{\mathrm{~d} t^{2}}+\left[\frac{\rho_{0} V}{2 \pi} \gamma_{2}-\gamma_{4}+2 \rho c W\right] \frac{\mathrm{d} T t}{\mathrm{~d} t}-N_{0 x} \frac{\partial^{2} W}{\partial x^{2}}+N_{0 y} \frac{\partial^{2} W}{\partial y^{2}}+\frac{\rho_{0} V^{2}}{2 \pi} \gamma_{3} T t\right. \\
& \left.-h \frac{\partial^{2} \Phi}{\partial y^{2}} \frac{\partial^{2} W}{\partial x^{2}}+\frac{\partial^{2} \Phi}{\partial x^{2}} \frac{\partial^{2} W}{\partial y^{2}} T^{3} t\right\} W x, y \mathrm{~d} x \mathrm{~d} y=0,
\end{aligned}
$$

where $S \in\{0 \leq x \leq a, 0 \leq y \leq b\}$.

where

Simplifying equation (26) yields

$$
A \frac{\mathrm{d}^{2} T(t)}{\mathrm{d} t^{2}}+B \frac{\mathrm{d} T(t)}{\mathrm{d} t}-C T(t)-D T^{3}(t)=0,
$$

$$
\begin{aligned}
& A=\iint_{S}\left(\rho W+\frac{\rho_{0}}{\pi} \gamma_{1}\right) W \mathrm{~d} x \mathrm{~d} y \\
& =\rho \iint_{S}\left(\sin \frac{m \pi x}{a} \sin \frac{n \pi y}{b}\right)^{2} \mathrm{~d} x \mathrm{~d} y+\frac{\rho_{0}}{\pi} \iint_{R a}\left(\iint_{R a} \frac{1}{r} \sin \frac{m \pi \xi}{a} \sin \frac{n \pi \eta}{b} \mathrm{~d} \xi \mathrm{d} \eta\right) \sin \frac{m \pi x}{a} \sin \frac{n \pi y}{b} \mathrm{~d} x \mathrm{~d} y \\
& =\frac{\rho a b}{4}+\frac{\rho_{0}}{\pi} \alpha_{1} \text {, } \\
& \alpha_{1}=\iint_{S}\left(\iint_{R a} \frac{1}{r} \sin \frac{m \pi \xi}{a} \sin \frac{n \pi \eta}{b} \mathrm{~d} \xi \mathrm{d} \eta\right) \sin \frac{m \pi x}{a} \sin \frac{n \pi y}{b} \mathrm{~d} x \mathrm{~d} y, \\
& B=\frac{\rho_{0} V}{2 \pi} \iint_{S}\left(\gamma_{2}-\gamma_{4}\right) W \mathrm{~d} x \mathrm{~d} y+2 \rho c \iint_{S} W^{2} \mathrm{~d} x \mathrm{~d} y \\
& =\frac{\rho_{0} m V}{2 a} \iint_{S}\left(\iint_{R a r} \frac{1}{r} \cos \frac{m \pi \xi}{a} \sin \frac{n \pi \eta}{b} \mathrm{~d} \xi \mathrm{d} \eta\right) \sin \frac{m \pi x}{a} \sin \frac{n \pi y}{b} \mathrm{~d} x \mathrm{~d} y \\
& -\frac{\rho_{0} V}{2 \pi} \iint_{S}\left[\iint_{R a} \frac{1}{r^{3}}(x-\xi) \sin \frac{m \pi \xi}{a} \sin \frac{n \pi \eta}{b} \mathrm{~d} \xi \mathrm{d} \eta\right] \sin \frac{m \pi x}{a} \sin \frac{n \pi y}{b} \mathrm{~d} x \mathrm{~d} y \\
& +2 \rho c \iint_{S}\left(\sin \frac{m \pi x}{a} \sin \frac{n \pi y}{b}\right)^{2} \mathrm{~d} x \mathrm{~d} y \\
& =\frac{\rho_{0} m V}{2 a} \alpha_{2}-\frac{\rho_{0} V}{2 \pi} \alpha_{4}+\frac{\rho c a b}{2} \text {, } \\
& \alpha_{2}=\iint_{S}\left(\iint_{R a} \frac{1}{r} \cos \frac{m \pi \xi}{a} \sin \frac{n \pi \eta}{b} d \xi d \eta\right) \sin \frac{m \pi x}{a} \sin \frac{n \pi y}{b} \mathrm{~d} x \mathrm{~d} y, \\
& \alpha_{4}=\iint_{S}\left[\iint_{R a} \frac{1}{r^{3}}(x-\xi) \sin \frac{m \pi \xi}{a} \sin \frac{n \pi \eta}{b} \mathrm{~d} \xi \mathrm{d} \eta\right] \sin \frac{m \pi x}{a} \sin \frac{n \pi y}{b} \mathrm{~d} x \mathrm{~d} y, \\
& C=\iint_{S}\left(N_{0 x} \frac{\partial^{2} W}{\partial x^{2}}+N_{0 y} \frac{\partial^{2} W}{\partial y^{2}}+\frac{\rho_{0} V^{2}}{2 \pi} \gamma_{3}\right) W \mathrm{~d} x \mathrm{~d} y \\
& =\iint_{S} N_{0 x} \frac{\partial^{2} W}{\partial x^{2}} W \mathrm{~d} x \mathrm{~d} y+\iint_{R a} N_{0 y} \frac{\partial^{2} W}{\partial y^{2}} W \mathrm{~d} x \mathrm{~d} y+\frac{\rho_{0} V^{2}}{2 \pi} \iint_{R a} \gamma_{3} W \mathrm{~d} x \mathrm{~d} y \\
& =-\frac{m^{2} \pi^{2} b N_{0 x}}{4 a}-\frac{n^{2} \pi^{2} a N_{0 y}}{4 b}+\frac{\rho_{0} m V^{2}}{2 a} \alpha_{3}, \\
& \alpha_{3}=\iint_{S}\left(\iint_{R a} \frac{1}{r^{3}}(x-\xi) \cos \frac{m \pi \xi}{a} \sin \frac{n \pi \eta}{b} d \xi \mathrm{d} \eta\right) \sin \frac{m \pi x}{a} \sin \frac{n \pi y}{b} \mathrm{~d} x \mathrm{~d} y, \\
& D=\iint_{S} h\left(\frac{\partial^{2} \Phi}{\partial y^{2}} \frac{\partial^{2} W}{\partial x^{2}}+\frac{\partial^{2} \Phi}{\partial x^{2}} \frac{\partial^{2} W}{\partial y^{2}}\right) W \mathrm{~d} x \mathrm{~d} y \\
& =-\frac{h m^{2} n^{2} \pi^{4}(\alpha+\beta)}{2 a b} \text {. }
\end{aligned}
$$




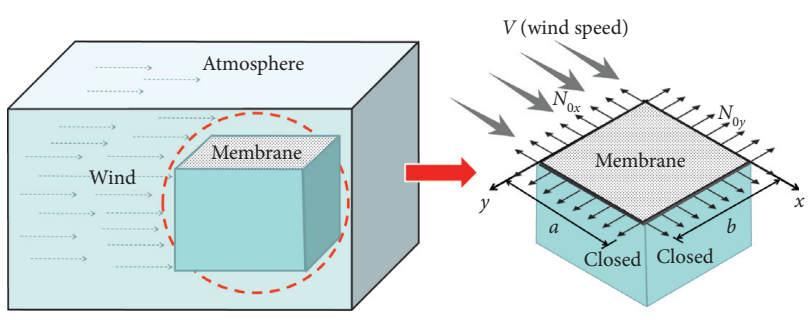

FIGURE 2: The membrane roof on closed structure.

It can be obtained from numerical calculation that $A \leq 0$ occurs only when $b / a \ll 1$, and this will not happen in practical engineering. Equation (27) is a strongly nonlinear vibration equation. For different equations of strongly nonlinear vibration, many scholars have studied the corresponding solutions [13-17]. In this paper, we propose the improved multiscale method based on the principle of modified Lindestedt-Poincaré method to solve equation (27). Letting $u=B /(\varepsilon A), \omega_{0}^{2}=-C / A, \varepsilon=-D / A$, then equation (27) can be transformed as

$$
\ddot{T}+\omega_{0}^{2} T+\varepsilon\left(\mu \dot{T}+T^{3}\right)=0 .
$$

Letting $\omega$ be the vibration frequencies of the membrane material and expanding $\omega^{2}$ to the power series of $\varepsilon$ near $\omega_{0}^{2}$ we obtain the following:

$$
\omega^{2}=\omega_{0}^{2}+\varepsilon \omega_{1}+\varepsilon^{2} \omega_{2}+\cdots
$$

The transformation parameters are introduced as follows:

$$
\begin{aligned}
\alpha & =\frac{\varepsilon \omega_{1}}{\omega_{0}^{2}+\varepsilon \omega_{1}}, \\
\varepsilon & =\frac{\omega_{0}^{2} \alpha}{\omega_{1}(1-\alpha)}, \\
\omega_{0}^{2}+\varepsilon \omega_{1} & =\frac{\omega_{0}^{2}}{1-\alpha} .
\end{aligned}
$$

Expand $\omega^{2}$ to the power series of $\varepsilon$ as follows:

$$
\begin{aligned}
\omega^{2} & =\omega_{0}^{2}+\varepsilon \omega_{1}\left[1+\frac{1}{\omega_{0}^{2}+\varepsilon \omega_{1}}\left(\varepsilon^{2} \omega_{2}+\varepsilon^{3} \omega_{3}+\cdots\right)\right] \\
& =\frac{\omega_{0}^{2}}{1-\alpha}\left(1+\delta_{2} \alpha^{2}+\delta_{3} \alpha^{3}+\cdots\right), \\
\omega & =\omega_{0}\left[1+\frac{1}{2} \alpha+\left(\frac{3}{8}+\frac{\delta^{2}}{2}\right) \alpha^{2}+\cdots\right] .
\end{aligned}
$$

The form of the perturbation solution of equation (29) can be

$$
T(t, \alpha)=T_{0}\left(t_{0}, t_{1}\right)+\alpha T_{1}\left(t_{0}, t_{1}\right)+\alpha^{2} T_{2}\left(t_{0}, t_{1}\right)+\cdots,
$$

where $t_{0}=t$ and $t_{1}=\alpha t$.

The differential operators are obtained as follows:

$$
\begin{aligned}
& \frac{\mathrm{d}}{\mathrm{d} t}=D_{0}+\alpha D_{1}+\alpha^{2} D_{2}+\cdots, \\
& \frac{\mathrm{d}^{2}}{\mathrm{~d} t^{2}}=D_{2}^{0}+2 \alpha D_{0} D_{1}+\alpha^{2}\left(D_{1}^{2}+2 D_{0} D_{2}\right)+\cdots
\end{aligned}
$$

Substituting equations (31)-(33) and (35) into equation (29) yields

$$
\begin{aligned}
& (1-\alpha)\left[D_{2}^{0}+2 \alpha D_{0} D_{1}+\alpha^{2}\left(D_{1}^{2}+2 D_{0} D_{2}\right)\right] \\
& \left(T_{0}+\alpha T_{1}+\alpha^{2} T_{2}+\cdots\right)+(1-\alpha) \omega_{0}^{2}\left(T_{0}+\alpha T_{1}+\alpha^{2} T_{2}+\cdots\right) \\
& +\frac{\alpha \omega_{0}^{2}}{\omega_{1}^{2}}\left[\left(T_{0}+\alpha T_{1}+\alpha^{2} T_{2}+\cdots\right)^{3}+\left(D_{0}+\alpha D_{1}+\alpha^{2} D_{2}\right)\right. \\
& \left.\cdot\left(T_{0}+\alpha T_{1}+\alpha^{2} T_{2}+\cdots\right)\right]=0
\end{aligned}
$$

$\alpha^{0}, \quad D_{0}^{2} T_{0}+\omega_{0}^{2} T_{0}=0$,

$\alpha^{1}, \quad D_{0}^{2} T_{1}+\omega_{0}^{2} T_{1}+2 D_{0} D_{1} T_{0}+\frac{\omega_{0}^{2}}{\omega_{1}}\left(D_{0} T_{0}+T_{0}^{3}\right)=0$,

$\alpha^{2}, \quad D_{0}^{2} T_{2}+T_{2}+2 D_{0} D_{1} T_{1}+\left(D_{1}^{2}+2 D_{0} D_{2}\right) T_{0}+\frac{\omega_{0}^{2}}{\omega_{1}} 3 T_{0}^{2} T_{1}=0$.

The solution of the first equation in the system of equation (38) can be obtained as

$$
T_{0}=A\left(t_{1}\right) e^{i \omega_{0} t_{0}}+\bar{A}\left(t_{1}\right) e^{-i \omega_{0} t_{0}} .
$$

Substituting equation (39) into the second equation of (38) yields

$$
\begin{aligned}
& D_{0}^{2} T_{1}+\omega_{0}^{2} T_{1}+\left(2 i \omega_{0} D_{1} A+3 \frac{\omega_{0}^{2}}{\omega_{1}} A_{2} \bar{A}+i \mu \frac{\omega_{0}^{3}}{\omega_{1}} A\right) e^{i t_{0}} \\
& +\frac{1}{\omega_{1}} A^{3} e^{3 i t_{0}}+c c=0,
\end{aligned}
$$

where $c c$ is the complex conjugate term. Eliminating the term of immortality in equation (40) yields

$$
2 D_{1} A+3 \frac{\omega_{0}}{\omega_{1}} A^{2} \bar{A}+i \mu \frac{\omega_{0}^{3}}{\omega_{1}} A=0 .
$$

Solving equation (41), we can obtain

$$
T_{1}=\frac{1}{8 \omega_{1}}\left(A^{3} e^{3 i t_{0}}+\bar{A}^{3} e^{-3 i t_{0}}\right) .
$$

Letting $A=1 / 2 f e^{i \phi}$, bring it into equation (42) and separat the imaginary part from the real part:

$$
\begin{aligned}
\frac{\mathrm{d} f}{\mathrm{~d} t_{1}} & =-\frac{1}{2} \mu \frac{\omega_{0}^{2}}{\omega_{1}} f, \\
f \frac{\mathrm{d} \phi}{\mathrm{d} t_{1}} & =-\frac{3 \omega_{0}}{8 \omega_{1}} f^{3} .
\end{aligned}
$$


Substituting $A=1 / 2 f e^{i \phi}$ into equation (39) yields

$$
T_{0}=f \cos \left(\omega_{0} t_{0}+\phi\right)=f \cos \left(\omega t+\phi_{0}\right) .
$$

Comparing the angular frequency in equation (34) with equation (44), we can get the equation under the first-order approximation condition as

$$
\frac{\mathrm{d} \phi}{\mathrm{d} t_{1}}=\frac{\omega_{0}}{2} .
$$

According to equations (43) and (44) yields

$$
\omega_{1}=\frac{3}{4} f^{2} \text {. }
$$

Substituting equation (46) into equation (43) and omitting higher order terms yields

$$
\omega=\sqrt{\omega_{0}^{2}+\frac{3}{4} \varepsilon f^{2}} .
$$

The results show that when the critical wind velocity of single-mode instability is reached, the frequency of the characteristic equation of the system approaches zero, which is equivalent to static equilibrium instability [11]. That is,

$$
\omega_{0}^{2}+\frac{3}{4} \varepsilon f^{2}=0
$$

where $f$ is the vibration amplitude of the membrane,

$$
\begin{aligned}
\omega_{0}^{2} & =\frac{2 \pi}{\rho a b \pi+4 \rho_{0} \alpha_{1}} \cdot\left(\frac{m^{2} \pi^{2} b^{2} N_{0 x}+n^{2} \pi^{2} a^{2} N_{0 y}}{2 a b}-\frac{\rho_{0} m \alpha_{3}}{a} V^{2}\right), \\
\alpha_{1} & =\iint_{S}\left(\iint_{R a} \frac{1}{r} \sin \frac{m \pi \xi}{a} \sin \frac{n \pi \eta}{b} \mathrm{~d} \xi \mathrm{d} \eta\right) \sin \frac{m \pi x}{a} \sin \frac{n \pi y}{b} \mathrm{~d} x \mathrm{~d} y, \\
\alpha_{3} & =\iint_{S}\left(\iint_{R a} \frac{1}{r^{3}}(x-\xi) \cos \frac{m \pi \xi}{a} \sin \frac{n \pi \eta}{b} \mathrm{~d} \xi \mathrm{d} \eta\right) \sin \frac{m \pi x}{a} \sin \frac{n \pi y}{b} \mathrm{~d} x \mathrm{~d} y, \\
\varepsilon & =\frac{2 h m^{2} n^{2} \pi^{5}(\alpha+\beta)}{a b\left(\rho a b \pi+4 \rho_{0} \alpha_{1}\right)} .
\end{aligned}
$$

Solving the equation, the critical wind velocity for singlemode instability of the closed membrane roof is obtained as follows:

$$
V_{c r}=\sqrt{\frac{4 \pi\left(m^{2} \pi^{2} b^{2} N_{0 x}+n^{2} \pi^{2} a^{2} N_{0 y}\right)+3 \varepsilon f^{2}\left(\rho \pi a_{2} b^{2}+4 \rho_{0} a b \alpha_{1}\right)}{8 \pi b \rho_{0} m \alpha_{3}}},
$$

where $f$ is the vibration amplitude corresponding to the instability critical wind velocity. It shows that the critical wind velocity is related to the vibration amplitude. With the consideration of the effect of geometric nonlinearity, the stiffness of the membrane varies with the amplitude in the process of vibration, which will affect the aerodynamic stability of the membrane roof to a certain extent. This conclusion is consistent with that of previous research studies $[9,10,18]$. When $f \longrightarrow 0$, the critical wind velocity of instability can be obtained according to the theory of small deflection $[9,10]$.

\subsubsection{Solution of Flexible Membrane Roof on Open Structure.} For membrane roofs on open structures, air flows on both sides of the membrane surface due to the smaller thickness of the membrane, which can be substantially determined by the thin-airfoil theory. The membrane roof on open structure is shown in Figure 3.
For the membrane roof on open structure, the aerodynamic force acting on the unit area of the membrane projection surface is expressed as [11]

$$
p=\rho_{0} \frac{\partial}{\partial t} \int_{0}^{x} \gamma_{c}(\xi, y, t) \mathrm{d} \xi+\rho_{0} V \gamma_{c}
$$

where $\gamma_{c}$ is the density of vortices:

$$
\gamma_{c}=a V \gamma_{j}=a V\left(a_{1 j} T(t)+a_{2 j} \frac{T^{\prime}(t)}{V}\right), \quad j=1,2, \ldots, M \times N .
$$

Substituting equation (52) into equation (9) yields

$$
\begin{aligned}
& \left(h \frac{\partial^{2} \varphi}{\partial y^{2}}+N_{0 x}\right) \frac{\partial^{2} w}{\partial x^{2}}+\left(h \frac{\partial^{2} \varphi}{\partial x^{2}}+N_{0 y}\right) \frac{\partial^{2} w}{\partial y^{2}}-2 \rho \xi_{0} \frac{\partial w}{\partial t} \\
& +\rho_{0} V \gamma_{c}+\rho_{0} \int_{0}^{y} \frac{\partial \gamma_{c}}{\partial t} \mathrm{~d} \eta=\rho \frac{\partial^{2} w}{\partial t^{2}} .
\end{aligned}
$$




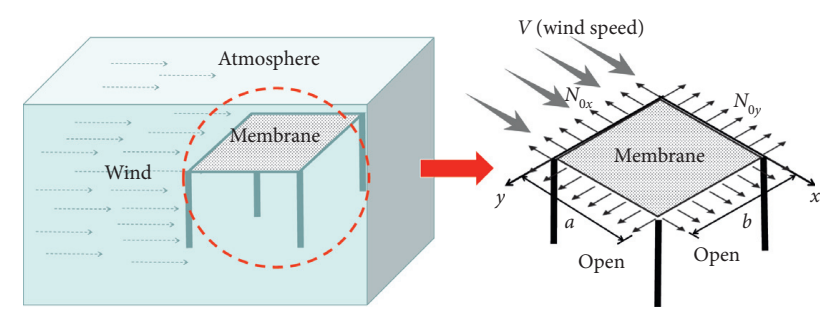

FIgURE 3: The membrane roof on open structure.

Solutions of stress functions in compatible equations such as equation (21). Substituting equations (19) and (21) into equation (53) yields

$$
\begin{aligned}
& \rho W \frac{\mathrm{d}^{2} T(t)}{\mathrm{d} t^{2}}+2 \rho \xi_{0} W \frac{\mathrm{d} T(t)}{\mathrm{d} t}-\left(N_{0 x} \frac{\partial^{2} W}{\partial x^{2}}+N_{0 y} \frac{\partial^{2} W}{\partial y^{2}}\right) T(t) \\
& -h\left(\frac{\partial^{2} \phi}{\partial y^{2}} \frac{\partial^{2} W}{\partial x^{2}}+\frac{\partial^{2} \phi}{\partial x^{2}} \frac{\partial^{2} W}{\partial y^{2}}\right) T^{3}(t)-\rho_{0} V \gamma_{c}-\rho_{0} \int_{0}^{y} \frac{\partial \gamma_{c}}{\partial t} \mathrm{~d} \eta=0 .
\end{aligned}
$$

By using the Bubnov-Galerkin method, (54) can be integrated as

$$
\begin{gathered}
\iint_{S}\left[\rho W \frac{\mathrm{d}^{2} T(t)}{\mathrm{d} t^{2}}+2 \rho \xi_{0} W \frac{\mathrm{d} T(t)}{\mathrm{d} t}-\left(N_{0 x} \frac{\partial^{2} W}{\partial x^{2}}+N_{0 y} \frac{\partial^{2} W}{\partial y^{2}}\right) T(t)\right. \\
\left.-h\left(\frac{\partial^{2} \phi}{\partial y^{2}} \frac{\partial^{2} W}{\partial x^{2}}+\frac{\partial^{2} \phi}{\partial x^{2}} \frac{\partial^{2} W}{\partial y^{2}}\right) T^{3}(t)-\rho_{0} V \gamma_{c}-\rho_{0} \int_{0}^{y} \frac{\partial \gamma_{c}}{\partial t} \mathrm{~d} \eta\right]
\end{gathered}
$$

$W(x, y) \mathrm{d} x \mathrm{~d} y=0$,

where $S \in\{0 \leq x \leq a, 0 \leq y \leq b\}$.

Integrating and simplifying equation (55) yields

$$
A \frac{\mathrm{d}^{2} T(t)}{\mathrm{d} t^{2}}+B \frac{\mathrm{d} T(t)}{\mathrm{d} t}-C T(t)-D T^{3}(t)-E=0,
$$

where

$$
\begin{aligned}
A & =\iint_{S} \rho W^{2} \mathrm{~d} x \mathrm{~d} y=\frac{\rho a b}{4} \\
B & =2 \rho \xi_{0} \iint_{S} W^{2} \mathrm{~d} x \mathrm{~d} y=2 \rho \xi_{0} \iint_{S}\left(\sin \frac{m \pi x}{a} \sin \frac{n \pi y}{b}\right)^{2} \mathrm{~d} x \mathrm{~d} y=\frac{\rho \xi_{0} a b}{2} \\
C & =\iint_{S}\left(N_{0 y} \frac{\partial^{2} W(x, y)}{\partial y^{2}}+N_{0 x} \frac{\partial^{2} W(x, y)}{\partial x^{2}}\right) W(x, y) \mathrm{d} x \mathrm{~d} y=\iint_{S} N_{0 x} \frac{\partial^{2} W}{\partial x^{2}} W \mathrm{~d} x \mathrm{~d} y+\iint_{S} N_{0 y} \frac{\partial^{2} W}{\partial y^{2}} W \mathrm{~d} x \mathrm{~d} y \\
& =-\frac{m^{2} \pi^{2} b N_{0 x}}{4 a}-\frac{n^{2} \pi^{2} a N_{0 y}}{4 b}, \\
D & =\iint_{S} h\left(\frac{\partial^{2} \Phi}{\partial y^{2}} \frac{\partial^{2} W}{\partial x^{2}}+\frac{\partial^{2} \Phi}{\partial x^{2}} \frac{\partial^{2} W}{\partial y^{2}}\right) W \mathrm{~d} x \mathrm{~d} y=-\frac{h m^{2} n^{2} \pi^{4}(\alpha+\beta)}{2 a b} \\
E & =\iint_{S}\left(\rho_{0} V \gamma+\rho_{0} \int_{0}^{y} \frac{\partial \gamma}{\partial t} \mathrm{~d} \eta\right) W(x, y) \mathrm{d} x \mathrm{~d} y .
\end{aligned}
$$

Substituting equation (52) into equation (56) yields

where

$$
A_{1} \frac{\mathrm{d}^{2} T(t)}{\mathrm{d} t^{2}}+B_{1} \frac{\mathrm{d} T(t)}{\mathrm{d} t}-C_{1} T(t)-D T^{3}(t)=0,
$$

$$
\begin{aligned}
A_{1}= & A-\rho_{0} a \iint_{S}\left(\int_{0}^{y} a_{2 j} d \eta\right) W(x, y) \mathrm{d} x \mathrm{~d} y, \\
B_{1}= & B-\rho_{0} a V \iint_{S}\left(\int_{0}^{y} a_{1 j} d \eta\right) W(x, y) \mathrm{d} x \mathrm{~d} y \\
& -\rho_{0} a V \iint_{S} a_{2 j} W(x, y) \mathrm{d} x \mathrm{~d} y, \\
C_{1}= & C+\rho_{0} a V^{2} \iint_{S} a_{1 j} W(x, y) \mathrm{d} x \mathrm{~d} y .
\end{aligned}
$$


The composition of equation (58) is consistent with the vibration control equation of closed membrane roof, and the solving process is consistent with the above, which is not discussed here. Thus, the expression of critical wind velocity for single-mode instability of open flexible membrane roof can be obtained as

$$
V_{c r}=\pi \sqrt{\frac{\left(m^{2} b N_{0 x} / 4 a\right)+\left(n^{2} a N_{0 y} / 4 b\right)+\left(3 h m^{2} n^{2} \pi^{2}(\alpha+\beta) f^{2} / 8 a b\right)}{\rho_{0} a(a b / M N) \sum_{j=1}^{M \times N} a_{1 j} \sin \left(m \pi x_{j} / a\right) \sin \left(n \pi y_{j} / b\right)}} .
$$

\section{Validation of Theoretical Model}

3.1. Verification Statement. Due to the limitation of the existing research funds, we have not carried out the aeroelastic experiment of the membrane roof at present. Therefore, we use the wind tunnel experiment results of the aeroelastic models of membrane structure in reference [19] to verify the theoretical calculation results in this paper. Later, we will do relevant experiments, and the experimental results will be discussed in our next paper.

3.2. Verification Results. The experimental model in reference [19] is a hyperbolic membrane structure with a small sag, with length of $416 \mathrm{~mm}$ and width of $325 \mathrm{~mm}$, as shown in Figure 4. The wind direction is along the length. Experimental model parameters are shown in Table 1 .

Uematsu et al. found in experiments that when the wind velocity exceeds a certain threshold, the test model will have a single-frequency vibration. At the same time, the amplitude of the pulsation displacement jumps and then decays. Then, the wind velocity corresponding to this amplitude is the critical wind velocity of a certain order of instability of the membrane structure. Based on this, the critical wind velocities of the first two orders of each model are finally obtained. Finally, the theoretical calculation value of the single-mode method of the open membrane roof is calculated by (60). Because the amplitude of the test jump point is incomplete and $f$ in (60) cannot be obtained, the theoretical result can only obtain the minimum critical wind velocity, as shown in Table 2.

It can be obtained from Table 2. The theoretical results are close to the experimental results, and the general variation of theoretical calculation results is in line with the experimental results, but the theoretical calculation results of different models are higher than the experimental results. The reason is that there is a curvature difference between the theoretically calculated flat model and the experimental model, which cause the aerodynamic forces on the surface of the membrane roof to be underestimated. Furthermore, the relative difference of the first-order critical wind velocity is greater than the second-order wind velocity, which indicates that the aerodynamic effect has a greater impact on the first-order mode shape of the membrane, which is consistent with existing research conclusions [20-22]. In summary, it shows that the theoretical calculation method in this paper has the good applicability for membrane structures.

\section{Analysis of the Effect of Geometric Nonlinearity on Critical Wind Velocity}

Assuming that the wind velocity is in the $X$ direction, $m=n=1, b=20 \mathrm{~m}, N_{0 x}=2 \mathrm{kN} / \mathrm{m}$, and $N_{0 y}=2 \mathrm{kN} / \mathrm{m}$. Then, the difference between the critical wind velocity with and without considering the geometric nonlinearity of membranes are discussed. Finally, the necessity of the consideration of the geometrical nonlinearity in the design of such structures is given. In this paper, the expression of critical wind speed is obtained by mathematical derivation, and it is related to the vibration amplitude of the membrane. Because the geometric nonlinearity of the membrane is considered, the stiffness of the membrane varies with the amplitude in the process of vibration, which will affect the aerodynamic stability of the membrane roof to a certain extent.

Define $\lambda$ is the ratio of cross-wind $(Y)$ span to alongwind $(X)$ span. The variation of critical wind velocity with vibration amplitude for two types of membrane roofs with different span ratios is shown in Figure 5.

Taking the membrane roof on the closed structure as an example and considering the geometric nonlinearity of the membrane, the critical wind velocity for single-mode instability of the closed membrane roof is obtained by solving the equation:

$$
V_{c r}=\sqrt{\frac{4 \pi\left(m^{2} \pi^{2} b^{2} N_{0 x}+n^{2} \pi^{2} a^{2} N_{0 y}\right)+3 \varepsilon f^{2}\left(\rho \pi a^{2} b^{2}+4 \rho_{0} a b \alpha_{1}\right)}{8 \pi b \rho_{0} m \alpha_{3}}} .
$$




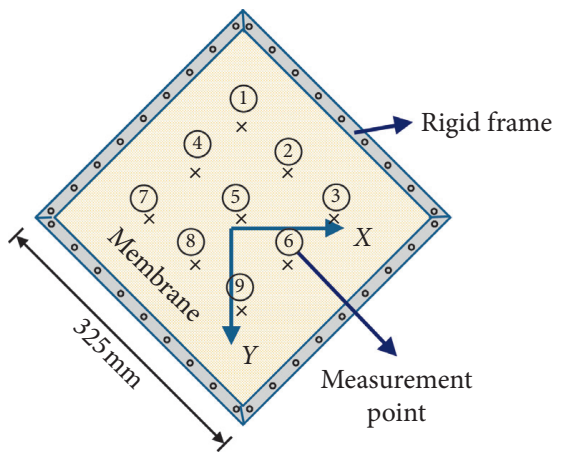

(a)

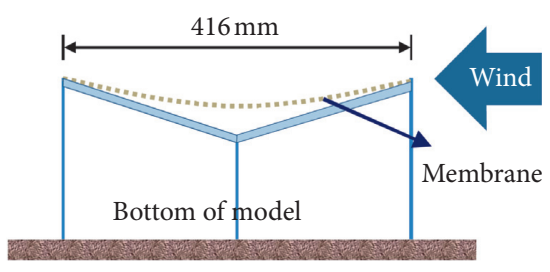

(b)

Figure 4: Experimental system.

TABle 1: Physical parameters of the experimental model.

\begin{tabular}{lccccccc}
\hline Model directory & $h(\mathrm{~mm})$ & $\rho\left(\mathrm{kg} / \mathrm{m}^{3}\right)$ & $E(\mathrm{MPa})$ & $\omega(\mathrm{Hz})$ & Damping ratio $(\%)$ & $N_{0 x}(\mathrm{~N} / \mathrm{mm})$ & $N_{0 y}(\mathrm{~N} / \mathrm{mm})$ \\
\hline B1 & 0.164 & 0.155 & 1.55 & 13.0 & 1.9 & 1.31 & 0.75 \\
B2 & 0.163 & 0.152 & 1.54 & 15.4 & 1.1 & 1.83 \\
B3 & 0.161 & 0.152 & 1.56 & 14.3 & 1.7 & 2.40 & 0.91 \\
B4 & 0.162 & 0.155 & 1.54 & 15.2 & 1.2 & 3.1 & 1.02 \\
\hline
\end{tabular}

TABLE 2: Comparison of theoretical calculation results with experimental results.

\begin{tabular}{lcccc}
\hline Model directory & Experimental results & \multicolumn{2}{c}{$\begin{array}{c}\text { Theoretical calculation } \\
\text { results }\end{array}$} & $V_{c r, s 1}(\mathrm{~m} / \mathrm{s})$ \\
\hline B1 & $V_{c r, t 1}(\mathrm{~m} / \mathrm{s})$ & $V_{c r, t 2}(\mathrm{~m} / \mathrm{s})$ & $>3.43$ & $>4.90$ \\
B2 & 3.0 & 4.7 & $>4.72$ & $>6.03$ \\
B3 & 4.1 & 5.9 & $>3.91$ & $>5.87$ \\
B4 & 3.5 & 5.5 & $>4.02$ & $>5.98$ \\
\hline
\end{tabular}

By substituting $f=0$ into equation (61), the results of critical wind velocity calculation without considering geometric nonlinearity can be obtained as

$$
V_{c r, L}=\pi \sqrt{\frac{a\left(\left(m^{2} b N_{0 x} / a\right)+\left(n^{2} a N_{0 y} / b\right)\right)}{2 \rho_{0} m \alpha_{3}}} .
$$

Comparing the critical wind velocity of the membrane roof on closed structure with and without considering the geometric nonlinearity, the following results are obtained:

$$
\frac{V_{c r}}{V_{c r, L}}(\text { closed })=\sqrt{1+\frac{3 \varepsilon f^{2}\left(\rho \pi a^{2} b^{2}+4 \rho_{0} a b \alpha_{1}\right)}{4 \pi\left(m^{2} \pi^{2} b^{2} N_{0 x}+n^{2} \pi^{2} a^{2} N_{0 y}\right)}}
$$

Similarly, we can also get the comparison results of membrane roofs on open structure as

$$
\frac{V_{c r}}{V_{c r, L}}(\text { open })=\sqrt{1+\frac{3 \varepsilon f^{2}\left(\rho \pi a^{2} b^{2}+4 \rho_{0} a b \alpha_{1}\right)}{4 \pi\left(m^{2} \pi^{2} b^{2} N_{0 x}+n^{2} \pi^{2} a^{2} N_{0 y}\right)}}
$$

Obviously, whether for the membrane roof on the closed structure or open structure, the ratio of critical wind velocity is the same with and without considering the geometric nonlinearity. Although compared with the open structure, the wall of the closed structure will affect the aerodynamic force of the membrane roof, but for the ratio of critical wind velocity equations (63) and (64), they are based on the same structure. Therefore, it is reasonable that the ratio of critical wind velocity of the closed structure and the open structure is the same.

\section{Discussion}

According to (63) and (64), whether for the membrane roof on the open structure or closed structure, $V_{c r} / V_{c r, L}>1$. So, the critical wind velocity of single-mode instability obtained with considering the geometrical nonlinearity of the membrane is larger than the linear one. The reason for this is that the stress increment, which is acquired from the geometric nonlinearity, can improve the lateral stiffness and enhance the aerodynamic stability of structures. It is conservative for structural design. By substituting the specific values, the ratio of critical wind velocity $V_{c r} / V_{c r, L}$ of the membrane roof on the closed structure can be obtained, as shown in Table 3. 


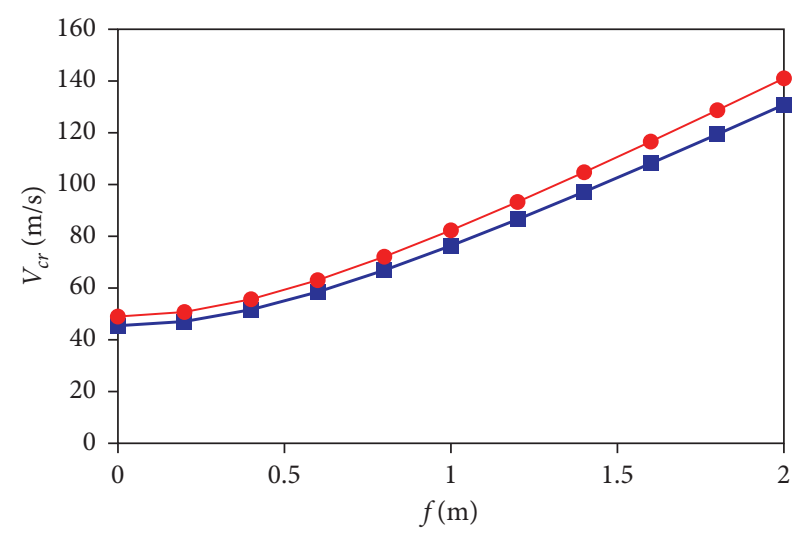

$\rightarrow-$ Membrane on closed structure

- Membrane on open structure

(a)

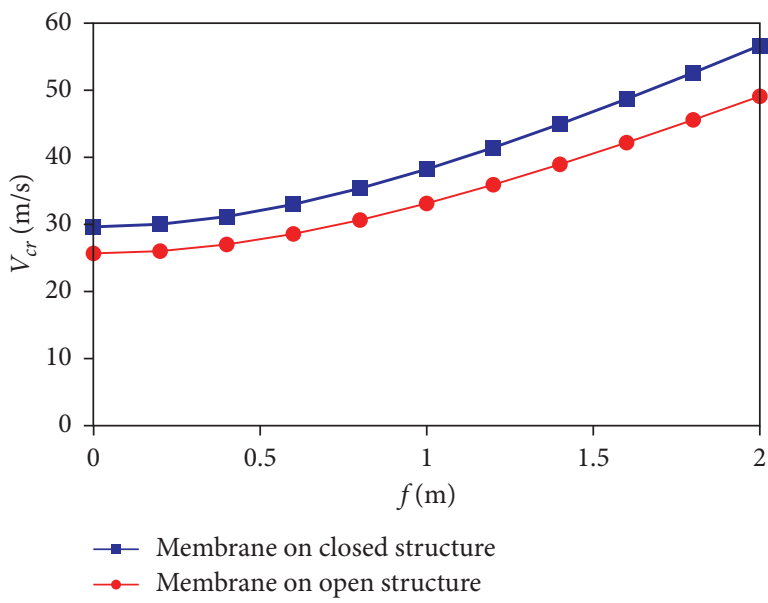

(b)

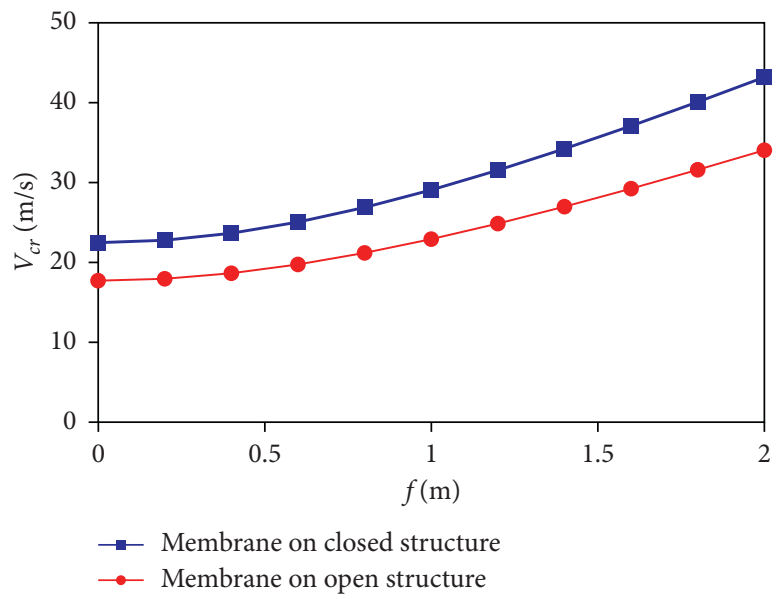

(c)

Figure 5: Curves of critical wind velocity with amplitude for two types roof models. (a) $\lambda=0.5$. (b) $\lambda=1$. (c) $\lambda=2$.

TABLE 3: Ratio of critical wind velocity of the membrane roof on the closed structure with and without considering geometric nonlinearity.

\begin{tabular}{lcccccccc}
\hline & $f=0.1 \mathrm{~m}$ & $f=0.2 \mathrm{~m}$ & $f=0.4 \mathrm{~m}$ & $f=0.6 \mathrm{~m}$ & $f=0.8 \mathrm{~m}$ & $f=1 \mathrm{~m}$ & $f=1.2 \mathrm{~m}$ & $f=1.4 \mathrm{~m}$ \\
\hline$\lambda=0.5$ & 1.00 & 1.04 & 1.14 & 1.29 & 1.47 & 1.68 & 1.90 & 2.14 \\
$\Lambda=1$ & 1.00 & 1.01 & 1.05 & 1.11 & 1.19 & 1.29 & 1.40 & 1.52 \\
$\Lambda=2$ & 1.00 & 1.01 & 1.05 & 1.11 & 1.20 & 1.29 & 1.40 & 1.52 \\
\hline
\end{tabular}

From Table 3, it can be seen that when the cross-wind span ratio is small. The geometric nonlinearity has a greater influence on the critical wind velocity of single-mode instability of the membrane roof, and the influence increases with the increase of the vibration amplitude. When the span ratio is greater than 1, the influence is relatively small. Article 5.3.4 of China's Technical Regulations for Membrane Structures (CECS 158:2015) [23] stipulates that for integral tensioned and cable-supported membrane structures, and the deformation of the membrane structure should not be larger than $1 / 200$ of the span when considering the combination of wind load effects. In the calculation example in this paper, the normal displacement is limited to $0.1 \mathrm{~m}$, and the ratio of critical wind velocity is 1.00 when geometric nonlinearity is considered or not. Therefore, the influence of membrane geometric nonlinearity on the aerodynamic stability of roofs can be neglected under normal wind loads. However, under strong wind loads, the deformation of roofs will exceed the norm limit. At this time, the influence of geometric nonlinearity should be considered.

\section{Conclusions}

In this paper, the aerodynamic stability of orthotropic rectangular planar membranes on closed and open structures is investigated by the mathematical analytic method. The governing equations of wind-induced nonlinear vibration of the tensioned membrane roofs are established by 
using the theory of large deflection of membrane and Darumbell's principle. The Bubov-Galerkin method is applied to transform the aerodynamically coupled governing equations into second-order constant coefficient nonlinear differential equations. Then, the periodic solution of the equation is calculated by using an improved multiscale method. Finally, critical wind velocity for single-mode instability of the membrane roof considering geometric nonlinearity is obtained by judging the stability of the periodic solutions of the equations. Furthermore, the influence of geometrical nonlinearity on the critical wind velocity of single-mode aeroelastic instability of membrane material is quantitatively obtained by comparing the results with those without consideration. The main conclusions can be summarized as follows:

(i) Considering the geometric nonlinearity of membranes vibration, the critical wind velocity of singlemode instability of membrane roof nonlinearly increases with the increase of transverse vibration displacement of membranes.

(ii) The critical wind velocity of single-mode instability with the geometrical nonlinearity of the membrane considered is larger than those of linear results. For structural design, it is conservative. When the span along the wind direction is small, the geometrical nonlinearity has a great influence on the critical wind velocity of single-mode instability of the membrane roof and with the amplitude of vibration. When the span ratio is greater than 1, there is no difference, as shown in Table 3. Only the vibration amplitude of 0.8 shows an insignificant.

(iii) Under normal wind loads, the influence of membrane geometric nonlinearity on the aerodynamic stability of roofs can be neglected. However, under strong wind loads, the deformation of roofs may exceed the norm limit and reach about $3 \%$ of the span, the influence of geometric nonlinearity should be considered.

\section{Abbreviations}

$a: \quad$ Length of the membrane roof

$b$ : Width of the membrane roof

$c$ : $\quad$ Viscous damping

$E_{1}$ : Young's modulus in $X$ direction

$E_{2}$ : $\quad$ Young's modulus in $Y$ direction

$f: \quad$ Amplitude of membrane

$h$ : Membrane's thickness

$N_{x}$ : The stress increment of membrane in $X$ direction

$N_{y}: \quad$ The stress increment of membrane in $Y$ direction

$N_{0 x}: \quad$ The initial stress of membrane in $X$ direction

$N_{0 y}$ : $\quad$ The initial stress of membrane in $Y$ direction

$p$ : $\quad$ Aerodynamic forces acting on the unit area of the membrane projection surface

$V: \quad$ Wind velocity

$V_{c r}$ : Critical wind velocity of divergence instability
$V_{c r, L}: \quad$ Critical wind velocity calculation without considering geometric nonlinearity

$W_{m n}(x, y)$ : Given mode shape function

$w(x, y, t): \quad$ Deflection of membrane

$z(x, y, t): \quad$ Membrane surface function under wind loadings

$z_{0}(x, y): \quad$ Membrane surface function under initial stress

$\varphi(x, y, t): \quad$ Stress function

$\gamma_{c}: \quad$ Density of vortices

$\lambda$ : $\quad$ Ratio of cross-wind $(Y)$ span to along-wind $(X)$ span

$\rho: \quad$ Areal density of membrane

$\rho_{0}: \quad$ Air density

$\omega$ : Vibration frequency of membrane.

\section{Data Availability}

The data used to support the findings of this study are available from the corresponding author upon request.

\section{Conflicts of Interest}

The authors declare that they have no conflicts of interest.

\section{Acknowledgments}

This research was funded by the National Natural Science Foundation of China (Grant no. 51608060) and the Scientific and Technological Research Program of Chongqing $\mathrm{Mu}$ nicipal Education Commission (Grant no. KJ1723378).

\section{References}

[1] C. H. Jenkins and J. W. Leonard, "Nonlinear dynamic response of membranes: state of the art," Applied Mechanics Reviews, vol. 49, no. 10S, pp. S41-S48, 1996.

[2] F. Rizzo and P. Zazzini, "Shape dependence of acoustic performances of buildings with a hyperbolic paraboloid cable net membrane roof," Acoustics Australia, vol. 45, no. 2, pp. 421-443, 2017.

[3] F. Rizzo, M. Barbato, and V. Sepe, "Peak factor statistics of wind effects for hyperbolic paraboloid roofs," Engineering Structures, vol. 173, pp. 313-330, 2018.

[4] Y. Wu, X. Y. Sun, and S. Z. Shen, "Computation of windstructure interaction on tension structures," Journal of Wind Engineering \& Industrial Aerodynamics, vol. 96, no. 10-11, pp. 2019-2032, 2008.

[5] A. Miyake, T. Yoshimura, and M. Makino, "Aerodynamic instability of suspended roof models," Journal of Wind Engineering and Industrial Aerodynamics, vol. 42, no. 1-3, pp. 1471-1482, 1992.

[6] Y. Wu and S. Z. Shen, "Research progress on fluid-solid interaction effect of wind-induced vibration response of membrane structure," Journal of Architecture and Civil Engineering, vol. 23, no. 1, pp. 1-9, 2006, in Chinese.

[7] Q. S. Yang and R. X. Liu, "On aerodynamic stability of membrane structures," Engineering Mechanics, vol. 23, no. 9, pp. 18-24, 2006, in Chinese.

[8] R. X. Liu and Q. S. Yang, "The wind-structure interaction equation of membrane roofs with small sag," Engineering Mechanics, vol. 21, pp. 41-44, 2004. 
[9] Y.-P. Xu, Z.-L. Zheng, C.-J. Liu, W.-J. Song, and J. Long, "Aerodynamic stability analysis of geometrically nonlinear orthotropic membrane structure with hyperbolic paraboloid," Journal of Engineering Mechanics, vol. 137, no. 11, pp. 759768, 2011.

[10] Z. L. Zheng, Y. P. Xu, and C. J. Liu, "Nonlinear aerodynamic stability analysis of orthotropic membrane structures with large amplitude," Structural Engineering \& Mechanics, vol. 37, no. 4, pp. 401-413, 2011.

[11] C. J. Liu, X. Deng, and Z. L. Zheng, "Nonlinear wind-induced aerodynamic stability of orthotropic saddle membrane structures," Journal of Wind Engineering and Industrial Aerodynamics, vol. 164, pp. 119-127, 2017.

[12] Z. L. Zheng, W. J. Song, and C. J. Liu, "Study on dynamic response of rectangular orthotropic membranes under impact loading," Journal of Adhesion Science and Technology, vol. 26, no. 10-11, pp. 1467-1479, 2012.

[13] K. Micallef, A. S. Fallah, P. T. Curtis, and L. A. Louca, "On the dynamic plastic response of steel membranes subjected to localised blast loading," International Journal of Impact Engineering, vol. 89, pp. 25-37, 2015.

[14] A. A. Babu, P. E. Sudhagar, and V. Rajamohan, "Dynamic characterization of thickness tapered laminated composite plates," Journal of Vibration and Control, vol. 22, no. 16, pp. 3555-3575, 2016.

[15] D. Balkan and Z. Mecitoğlu, "Nonlinear dynamic behavior of viscoelastic sandwich composite plates under non-uniform blast load: theory and experiment," International Journal of Impact Engineering, vol. 72, pp. 85-104, 2014.

[16] D. Li, Z. L. Zheng, R. Yang, and P. Zhang, "Analytical solutions for stochastic vibration of orthotropic membrane under random impact load," Materials, vol. 11, no. 7, pp. 1231-1259, 2018.

[17] Y. Y. Zhang and Q. L. Zhang, "Load-dependent mechanical of membrane materials and its effect on the static behaviors of membrane structures," Journal of Materials in Civil Engineering, vol. 51, pp. 468-473, 2015.

[18] Z. Q. Chen, Y. Wu, and X. Y. Sun, "Research on aeroelastic instability mechanism of closed-type one-way tensioned membrane," Journal of Building Structures, vol. 36, pp. 12-19, 2015.

[19] Y. Uematsu and K. Uchiyama, "Aeroelastic behavior of an H.P.-shape suspend rood," in Proceedings of the 1986 IASS Symposium on Membrane Structures and Space Frame, no. 2, pp. 241-248, Osaka, Japan, September 1986.

[20] Y. Q. Li, L. Wang, Z. Y. Shen, and Y. Tamura, "Added-mass estimation of flat membranes vibrating in still air," Journal of Wind Engineering and Industrial Aerodynamics, vol. 99, no. 8, pp. 815-824, 2011.

[21] Q. S. Yang, Y. Wu, and W. L. Zhu, "Experimental study on the static interaction between membrane structures and air," China Civil Engineering Journal, vol. 41, no. 5, pp. 19-25, 2008, in Chinese.

[22] W. J. Song, Y. M. Li, and X. W. Wang, "Study on the vibration of membrane roof with the influence of added air-mass," Journal of Physics Conference Series, vol. 1064, Article ID 012054, 2018.

[23] China Association for Engineering Construction Standardization, Technical Specification for Membrane Structures, China Planning Press, Beijing, China, 2015. 